\title{
Enhanced Acoustic Separation of Oil-Water Emulsion in Resonant Cavities
}

\author{
A. Garcia-Lopez ${ }^{*}$ and D.N. Sinha
}

Los Alamos National Laboratory, MPA-11, MS D429, Los Alamos, NM 87545, USA

\begin{abstract}
This paper explores the use of acoustics for the recovery of oil in oil-water emulsions. A series of experimental studies were conducted using acoustic standing waves in resonant cavities as means of trapping oil droplets and enhancing oil separation. Several cavity configurations were explored and the frequency range used was between $1 \mathrm{MHz}-2 \mathrm{MHz}$. Oil-water emulsions studied were made using mineral oil and motor oil.
\end{abstract}

\section{INTRODUCTION}

Acoustic standing waves have been used to manipulate solid particle-fluid systems in tubes and capillaries for various applications. The exploitation of acoustic technologies has been well established in the area of particle manipulation, e.g., particle transport, separation, or removal [1]. In contrast to the extensive amount of research in particle-fluid systems, the use of acoustic technology for liquid-liquid systems, such as emulsions has been focused mainly toward fundamental studies of nucleation [2], coagulation and dispersion [3], and coalescence [4]. The recovery of oil from oil-water emulsions is an important area of technology relevant to the petroleum industry. In recent years, there has been various technology developments in this area such as the use of alternating electric fields in electrical separation of oil [5]. In the area of acoustics, Pangu and Feke have demonstrated oil recovery from an aqueous emulsion using ultrasonic standing waves in a cavity with a porous material inside the cavity [6]. However this work was limited to a single cavity geometry and a single frequency and the oil used was vegetable oil. Crum [7] used a combination of ultrasound and electrolysis techniques to flocculate micelles and then coalescing the micelles for oil recovery. In the context of oil recovery from oil-water emulsions, the application of acoustics is rather limited.

The characteristics of the oil-water emulsion vary with its rheology that is dependant on temperature, pressure, particleparticle interaction and gravitational forces [8]. Obstacles to overcome in physically separating these emulsions using ultrasound are: stability of the emulsion (surfactant), the concentration of oil, and size of oil droplets. The objective of this paper is to present the results of an experimental study of oil droplet coalescence and separation in a couple of oilwater emulsions in different types of resonant cavities and the effect of a range of different resonance frequencies, acoustic power, and electrical signal waveforms. Our goal has been to understand heavy crude oil-water emulsion separation.

*Address correspondence to this author at the Los Alamos National Laboratory, MPA-11, MS D429, Los Alamos, NM 87545, USA;

E-mail: acgl@lanl.gov

\section{THEORY}

Ultrasonic standing waves can be generated in a fluidfilled cell if the applied ultrasonic frequency on opposite ends of the cell is tuned to satisfy the condition

$L=n \frac{\lambda}{2}(\mathrm{n}=1,2,3 \ldots)$

where, $L$ is the pathlength in the fluid and $\lambda$ the wavelength of the ultrasonic wave. In this case displacement nodes appear at the spacing of $\lambda / 2$. At the same time, pressure nodes appear at the positions of the displacement antinodes. Fluid containing small compressible spheres suspended in such a standing acoustic wave field experience various radiation forces. This force has three separate components: (1) primary axial acoustic radiation force $\left(F_{p}\right),(2)$ primary transverse acoustic radiation force, and (3) secondary acoustic radiation force $(F s)$. When the fluid is a two-phase system (e.g., an emulsion) the droplets of one phase in a second continuous host medium experience a time averaged primary axial acoustic force, which is generated by the interaction between droplets and the primary wave field. The primary acoustic force exerted on a droplet has been studied theoretically by many researchers [9-14]. For a compressible liquid drop this primary radiation force can be expressed as

$F_{p}=-4 \pi R^{3} k E_{a c} \cdot \phi(\beta, \rho) \cdot \sin \left(\frac{4 \pi z}{\lambda}\right)$

where $E_{a c}$ is the energy density of the acoustic field, $z$ is the distance from a pressure node, $R$ is the radius of the drop, and $k$ is the wave number of the driving frequency in the host liquid. The contrast factor $\phi$ is

$\phi(\beta, \rho)=\frac{5 \rho_{p}-2 \rho_{f}}{2 \rho_{p}+\rho_{f}}-\frac{\beta_{p}}{\beta_{f}}$

For liquids, the compressibility is expressed as $\beta=1 / \rho c^{2}$ where $c$ is the sound velocity of the liquid. In case $\phi$ takes a negative value, $F$ becomes the restoring force around the displacement node $(z=0)$; i.e., $F>0$ for $z<0$ and $F<0$ for $z>0$. Consequently, the droplet is trapped at the displacement node. On the other hand, if $\phi$ takes a positive value, $F$ becomes the restoring force around the displacement antinode 
or the pressure node $(\lambda / 4)$, and hence the droplet is trapped at the pressure node.

The acoustic contrast factor $\phi$ was calculated for mineral oil and motor oil in water using literature values of sound speed and density [15]. The literature values used for water were $0.999 \mathrm{~g} / \mathrm{cc}$ and the corresponding sound speed at 1480 $\mathrm{m} / \mathrm{s}$. Table 1 provides the contrast factor calculated using eq. (2) for mineral oil/water and motor oil/water.

Table 1. Acoustic Contrast of Mineral and Motor Oil

\begin{tabular}{|c|c|c|c|}
\hline & $c(\mathbf{m} / \mathbf{s})$ & $\rho\left(\mathbf{g} / \mathbf{c m}^{3}\right)$ & $\phi$ \\
\hline \hline Mineral Oil & 1430 & .821 & -0.53 \\
\hline Motor Oil & 1700 & .875 & -0.04 \\
\hline
\end{tabular}

When the droplets move closer to the pressure antinodes (pressure node for positive contrast factor), within a few diameters of another droplet, a secondary radiation force that is an attractive force between two spheres in an acoustic field, start to dominate. These interparticle forces drive the droplets together. Smaller droplets become larger droplets, and since the acoustic force is proportional to the particle volume ; it can grow rapidly. The secondary radiation force can be expressed as [16],

$F_{s}=-\frac{\kappa^{2} E_{a c}}{2 \pi}\left(1-\frac{\beta_{p}}{\beta_{f}}\right)^{2} \frac{V_{1} V_{2}}{d^{2}}$

where $V_{l}$ and $V_{2}$ are the volumes of the interacting droplets, $d$ is the separation distance between the centers of the droplets. As the droplets move through the liquid under the influence of the various acoustic forces mentioned above they experience hydrodynamic drag which is given by the drag force $F_{d}$ [17]

$F_{d}=-4 \pi\left(\frac{1+2 \hat{\mu} / 3}{1+\hat{\mu}}\right) \mu R V^{o}$

where $\mu$ is the viscosity of the fluid, $\hat{\mu}$ is the ratio of viscosity of the drop to the continuous phase and $V^{\circ}$ is the speed of the drop. Table 2 lists viscosity values at $40^{\circ} \mathrm{C}$ for water, light mineral oil, and 10W-40 motor oil for comparison. Motor oil is an order of magnitude more viscous than mineral oil. Consequently, motor oil takes somewhat longer to separate for the same values of applied power.

Table 2. Viscosity Values

\begin{tabular}{|c|c|c|c|}
\hline & Water & Mineral Oil & Motor Oil \\
\hline \hline Viscosity (cP@ $\left.@ 40^{\circ} \mathrm{C}\right)$ & 0.65 & 27.5 & 319 \\
\hline
\end{tabular}

In addition to the interdroplet force, the droplets also experience a radiation force that acts in the direction perpendicular to the direction of the sound beam which is horizontal in the our case. Although this force is estimated to be almost two orders of magnitude smaller than the primary radiation force, it is sufficient to induce clumping of the droplets [18-21]. This force counters the buoyancy force of the droplets.

Although there are plenty of models available to predict the acoustic behavior of oil drop movement in water; there does not exist a model to determine practical implementation of acoustic separation of oil and water for industry use. An objective of this study was to determine if acoustic separation could be performed on stable heavy crude oil-water emulsions. This is a particularly challenging problem because the density difference between water and heavy crude oil is very small. This paper explores the pragmatic performance of oil-water separation experimentally in simple resonant cavities without any artifacts in it by exploring two parameters aforementioned, transducer area by way of shape and frequency.

\section{MATERIALS}

All the experiments conducted use Stanford Research System $30 \mathrm{MHz}$ vs 345 function generator, ENI 310L RF power amplifier, Tektronix oscilloscope, Alan Industries 50 V10 attenuator, and Panametric piezo transducers of center frequencies $1 \mathrm{MHz}, 1.5 \mathrm{MHz}, 2.25 \mathrm{MHz}$. Oil-water emulsions were prepared using an Omni-Ruptor $250 \mathrm{Ho}-$ mogenizer. Oils used for the emulsion were Mineral oil and Pennzoil 10W40 motor oil. The motor oil emulsions were made with the surfactant Triton-x100 (phenol) for stabilization. An HP 4194A impedance analyzer was used for the electrical characterization of the transducers used and to determine the appropriate frequency sweep range.

\section{MINERAL OIL-WATER EMULSION EXPERIMENTS}

The emulsions used in these experiments were made of $10 \%$ by volume mineral oil in water prepared using the homogenizer. Three different shaped containers were used to setup standing waves in the emulsion: a rectangular quartz Starna (Starna Cells Inc.) cuvette, a cylindrical transducer, and a curved bottom cuvette. The frequency spectra obtained from frequency sweep measurements of the containers with piezoelectric transducers attached on the outside were composite spectra of the fluid-filled cavity and the container wall. Maximum sound transmission into the cavity occurred at the thickness mode resonance frequency of the container wall that is dependent on the material and thickness of the container wall. Therefore, resonant frequency appropriate for each container was used to set up standing waves for the experiment that provided the maximum energy into the cavity. Several transducer arrangements were tested using the rectangular cuvette. The first transducer arrangement used two transducers that were placed on opposite walls of the vertical cuvette $(10 \mathrm{~mm} \times 10 \mathrm{~mm} \times 45 \mathrm{~mm})$ filled with the emulsion. Initially, one transducer was used as the source

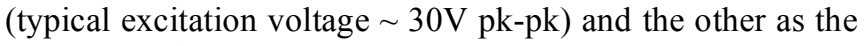
receiver to detect the resonance condition (standing wave) in the cavity. The cavity had several resonance frequencies between $1-2 \mathrm{MHz}$ and tests were performed at each of these frequencies. At each resonance frequency, mineral oil was observed to separate from the emulsion and form lines inside of the cuvette as in Fig. (1). A second transducer setup was 
tested which involved connecting both transducers to the power amplifier and placing an adjustable attenuator on one transducer. This was done to observe the effects of applying power from opposite sides of the cavity to modify the standing wave pattern while varying the power on one transducer and keeping the other fixed. The frequency was set to $1.345 \mathrm{MHz}$ at $30 \mathrm{~V}$ pk-pk and the attenuator was adjusted between $1-10 \mathrm{~dB}$. In each case, regardless of the signal attenuation, the mineral oil collected as lines in the cuvette by the standing waves produced. In all of the experiments previously described, once the power to the source transducer was turned off the oil could be seen rising to the top of the cuvette rapidly due to gravity. In an attempt to accelerate the rate at which the oil rose to the top, another transducer was placed at the bottom of the cuvette. The bottom transducer was excited at $49 \mathrm{kHz}$ with an excitation voltage of $19.2 \mathrm{~V}$ pk-pk using a separate function generator and amplifier. The frequency was chosen somewhat arbitrarily. Using the setup with the phase inverter all three transducers were turned on at the same time and after a few seconds only the transducers on the vertical walls were turned off. The presence of the excitation from below decreased the time of oil rising by half as compared to no additional excitation.

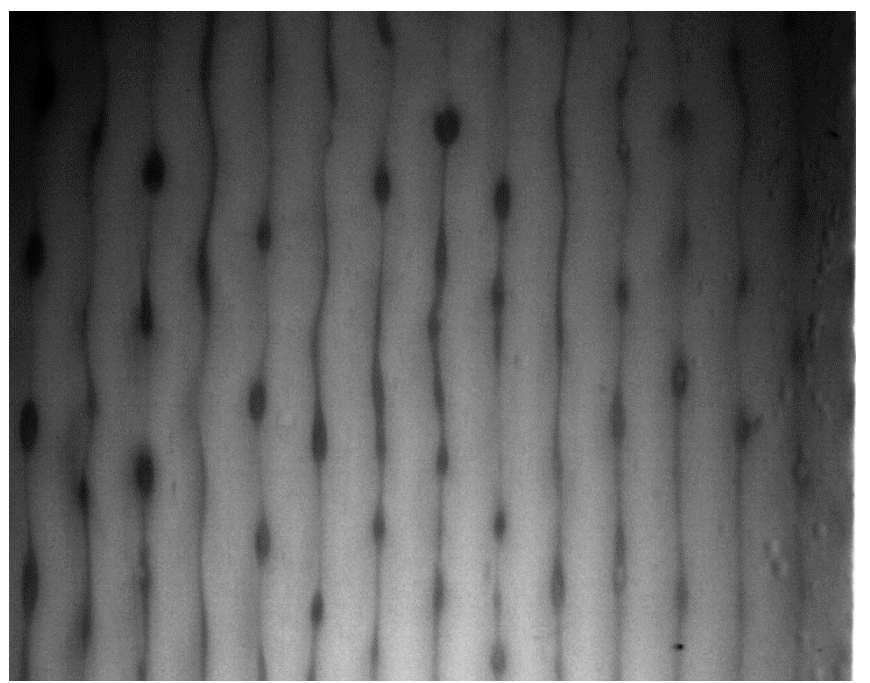

Fig. (1). Oil droplets captured in standing waves at $1.345 \mathrm{MHz}$ in quartz Starna cuvette.

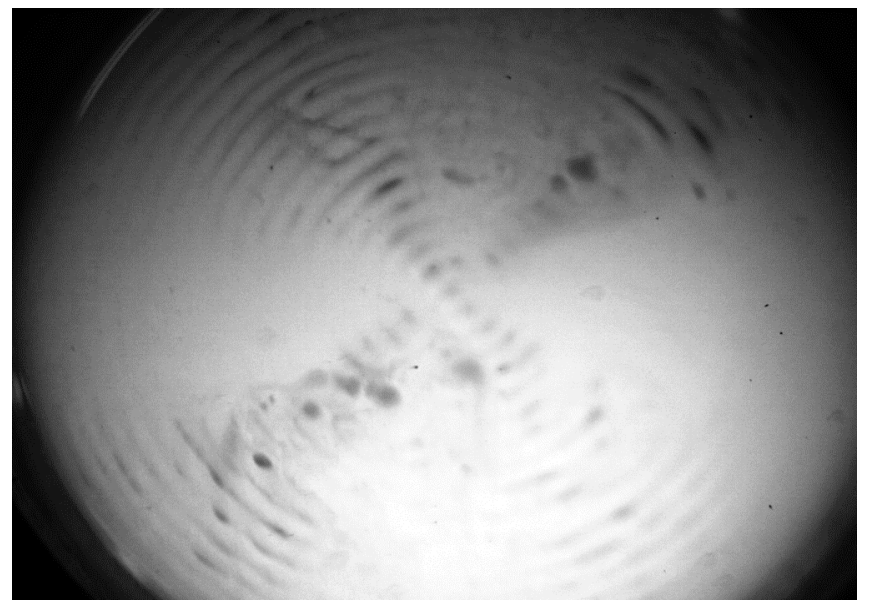

Fig. (2). Oil droplets captured in standing waves concentrically at $1.67 \mathrm{kHz}$ frequency modulated in cylindrical piezo transducer.

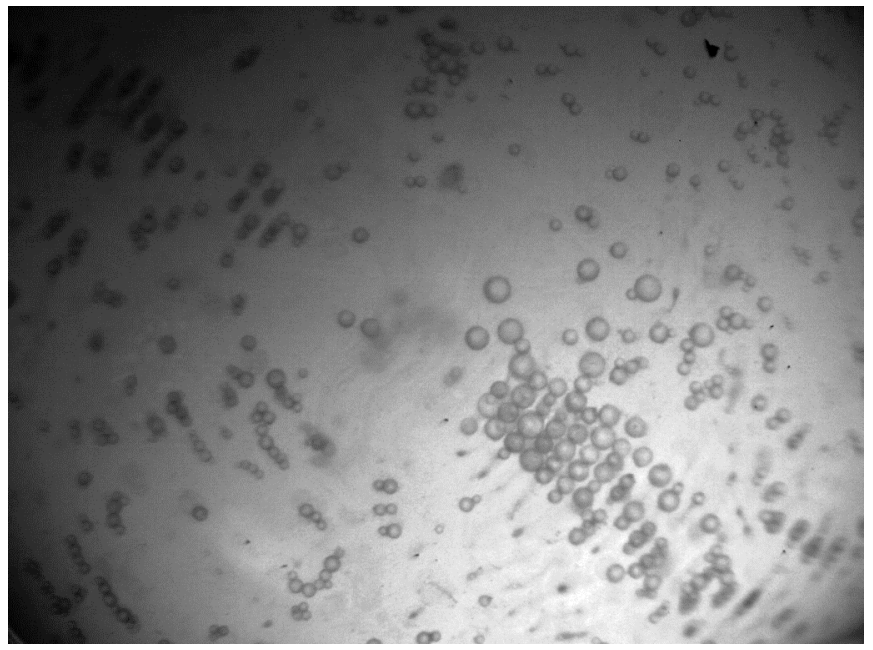

Fig. (3). Large oil droplets forming at $1.84 \mathrm{MHz}$ in cylindrical piezo transducer.

Following the experiments in containers, a hollow piezoelectric cylindrical transducer of dimensions: $20 \mathrm{~mm}$ height, $18 \mathrm{~mm}$ diameter, and $1.09 \mathrm{~mm}$ thickness with both inner and outer surface coated with an electrode material was tested to determine if direct contact with the transducer instead of the sound going through the wall of a cuvette enhanced the separation of an oil-water emulsion. The cylindrical transducer was converted into a container by placing a glass slide on the bottom side. The emulsion was poured into this cylindrical container and the signal from the function generator at $2.152 \mathrm{MHz}$ and $6 \mathrm{~V}$ pk-pk was applied directly to the outer and inner electrodes of the piezo-cylinder. This frequency was in the neighborhood of the thickness mode resonance frequency of the piezo-cylinder and corresponded to one of the cavity resonances. Standing waves of oil in circular patterns as concentric rings were observed because of the circular geometry of the container. Both frequency and amplitude modulations of the excitation signal were used in this setup as a means of controlling the power output and for forcing oil droplets to coalesce more efficiently. The effect of amplitude modulation was studied first; here clear images of concentric standing waves were formed at $1.841 \mathrm{MHz}$ (one of the cavity resonances) at $1 \mathrm{kHz}$ modulating frequency, as shown in Fig. (2). Frequency modulation was tested at 2.122 $\mathrm{MHz}$ with a $6 \mathrm{kHz}$ carrier wave at $30 \mathrm{~V} \mathrm{pk-pk}$. The frequency modulation showed an increase of oil droplet coalescence at the surface of the emulsion and while forming concentric standing waves (Fig. 3).

The standing waves produced previously were spaced evenly with the spacing determined by the resonant frequency. To produce standing waves at a focal point, and thus intensify the acoustic field, a cuvette was constructed using half of a hollow cylindrical piezo transducer. This U-shaped piezo with its axis horizontal was used as the bottom of the cuvette and glass rectangles were adhered to make the walls, resulting the dimensions of $24 \mathrm{~mm} \times 25 \mathrm{~mm} \times 35 \mathrm{~mm}$. The air-water interface was used as a reflecting surface for producing acoustic standing waves. Standing wave patterns of oil were visibly produced in the $10 \%$ mineral oil emulsion at 2.173 $\mathrm{MHz}$ and $2.25 \mathrm{MHz}$ set to $15.8 \mathrm{~V}$ pk-pk (Fig. 4). 


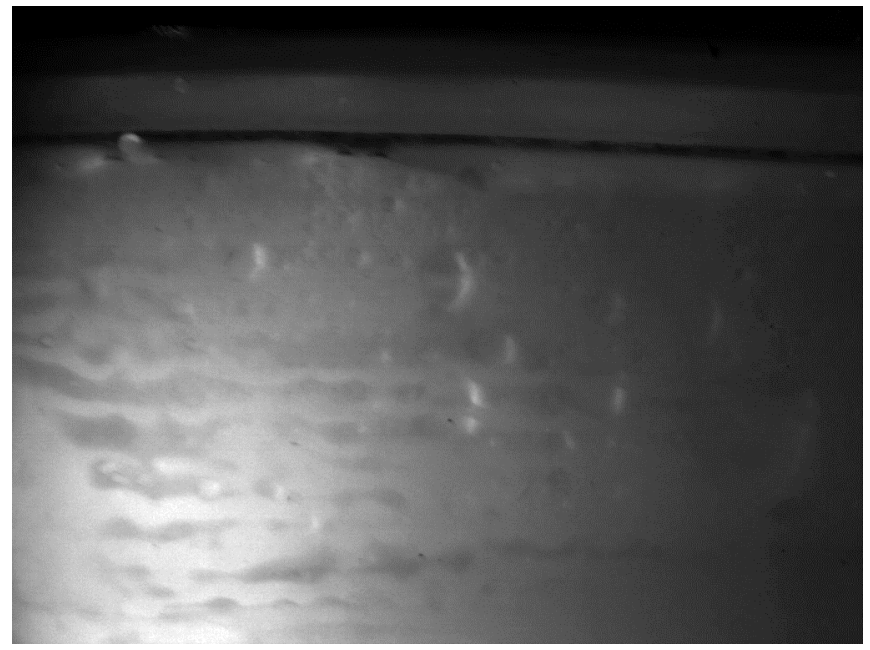

Fig. (4). Trapped oil in $2.173 \mathrm{MHz}$ curved cuvette

\section{MOTOR OIL-WATER EMULSION EXPERIMENTS}

In recovering oil from an oil-water emulsion, different viscosities of oil need to be considered. Motor oil 10W40water emulsions were made using $1 \%$ by volume of surfactant in water. To determine the effectiveness of the surfactant and the state of these emulsions; the speed of sound was measured for varying amounts of oil in water. The sound speed was determined using a frequency sweep technique under ambient conditions in a rectangular cuvette [22].

Measurements were made at $5 \%, 10 \%, 15 \%, 20 \%$, and $25 \%$ motor oil by volume emulsions as well as pure water and motor oil. The speed of sound was compared to the Urick model,

$v=\frac{1}{\sqrt{\rho_{m}^{2} \kappa_{m}}}$

$\rho_{m}=\phi \rho^{\prime}+(1-\phi) \rho$

$\kappa_{m}=\phi \kappa^{\prime}+(1-\phi) \kappa$

where $\kappa^{\prime}$ is the compressibility of oil and $\kappa$ is the compressibility of water, and $\phi$ the volume fraction. Meng et al. [23] showed that Urick Model is adequate for prediction of speed of sound in crude oil-water emulsion. The Urick model assumes the suspended particles are infinitesimally small compared to the wavelength of sound, and the effects of scattering neglected. Density and compressibility are averages weighted by phase concentration. As expected, the emulsion experiment values closely follow the Urick model, establishing that the starting condition is an emulsion instead of a stirred mixture (Fig. 5).

Measurements with motor-oil emulsions were very similar to that of mineral oil and the results were identical. To enhance the strength of the standing waves, an aluminum reflector was used instead of the previous air-water interface and it indeed produced a very strong standing wave pattern (Fig. 6).

The cylindrical container used in the mineral oil experiments was modified for the motor oil emulsion for visibility. An acrylic cylindrical container was fabricated and a cylin- drical transducer was placed inside the acrylic holder, as shown in Fig. (7). The emulsion was poured into the cylinder where some of the emulsion was in the concentric region between the container and transducer. The emulsion was

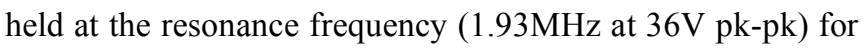
2 minutes. Standing waves were seen forming during the insonification process (Fig. 8). Once the power was turned off and the transducer removed, an oil layer could be seen forming at the top of the emulsion immediately.

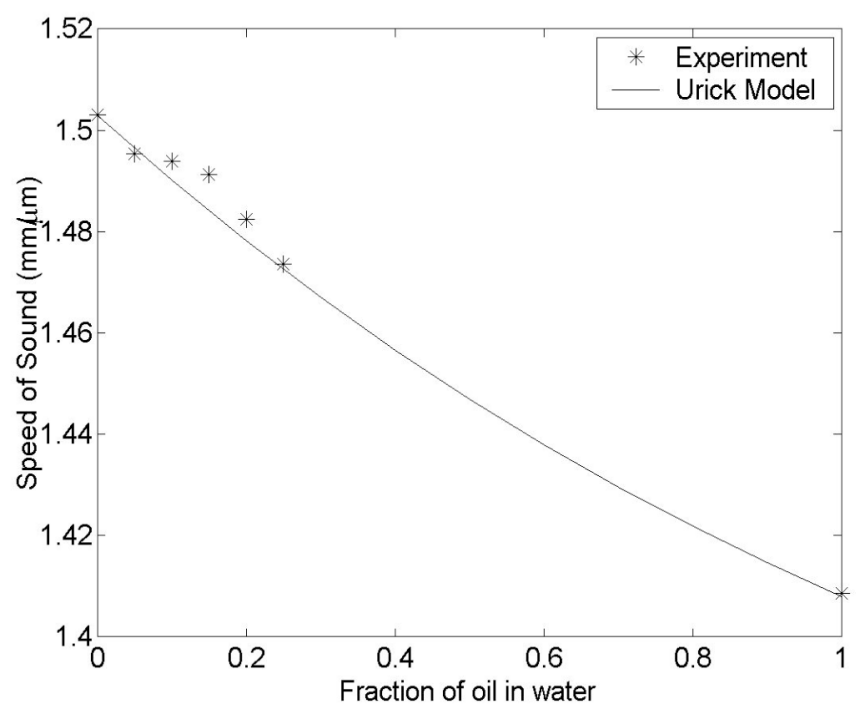

Fig. (5). Fraction of Motor oil in water vs speed of sound.

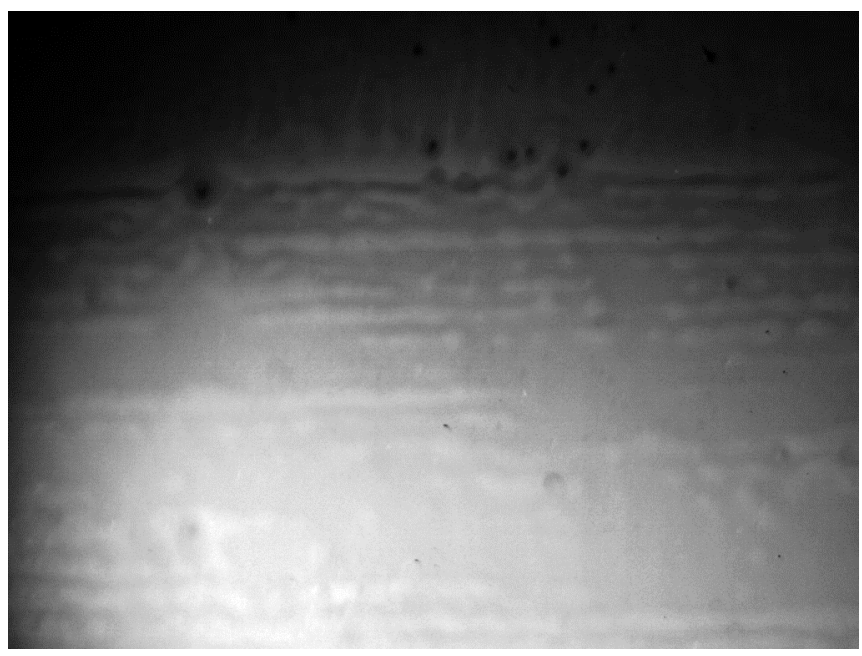

Fig. (6). Motor oil emulsions; lines are motor oil trapped in standing waves at $2.253 \mathrm{MHz}$ in curved cuvette.

Following this experiment a separate two acrylic container holder was made to study the difference in oil accumulation with and without acoustic exposure. Equal amounts of motor oil emulsion were placed into each of the containers and the piezo transducer was placed inside one of the containers. A downward ramped frequency sweep at $22 \mathrm{~V}$ pk-pk was sustained for 1, 2, 3 and 4 minutes. The results showed that over the prolonged exposure the oil collected at the top of the liquid was significantly more for the acoustically treated emulsion than without (Fig. 9). Comparing amplitude modulated, ramped frequency and static frequency at residence time of $4 \mathrm{~min}$ with the same conditions as before; all 


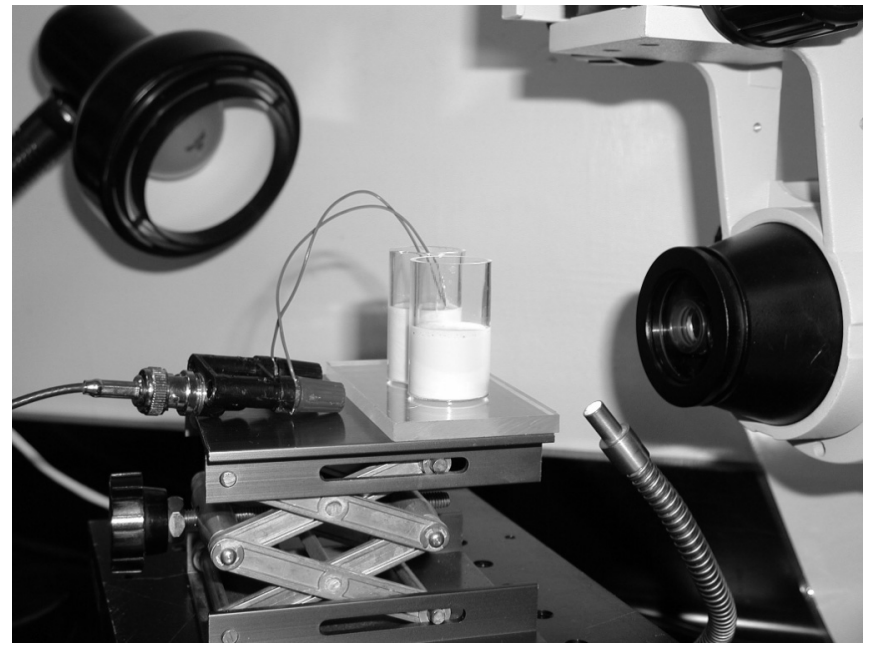

Fig. (7). Experimental setup.

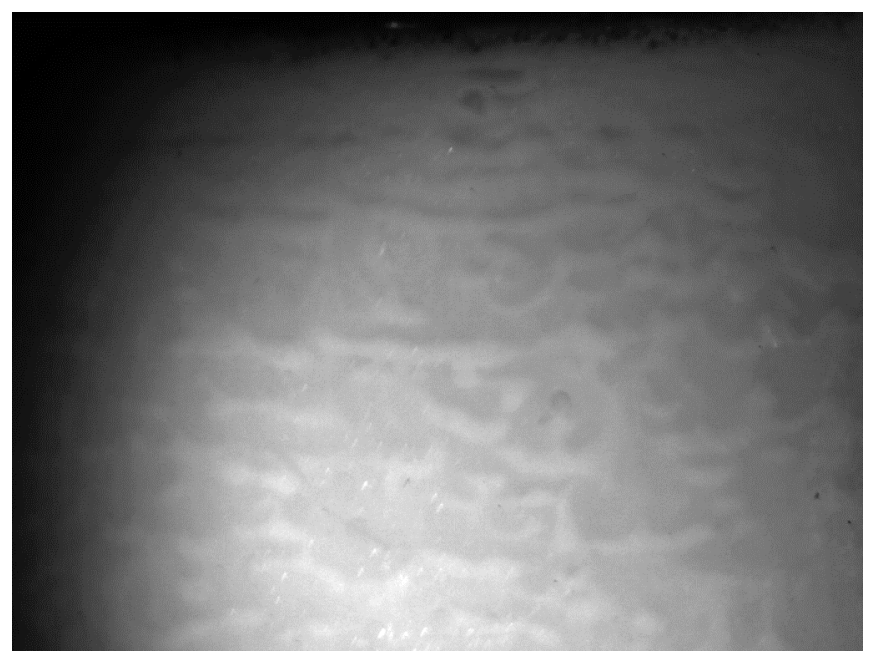

Fig. (8). Motor oil trapped in standing waves in cylindrical holder sandwiched by piezo cylinder transducer.

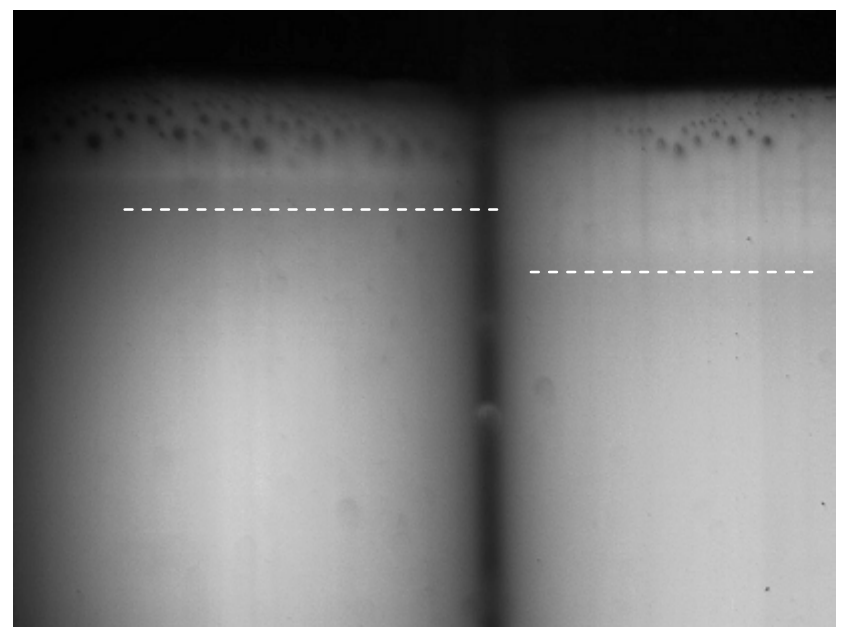

Fig. (9). Control on the left and experiment on the right showing motor oil levels recovered after 4 min of having applied acoustic field.

the results showed a significant increase in oil accumulation compared to the control, however when compared to one another all three experiments looked the same in terms of oil layer thickness. A power level experiment was conducted where for $4 \mathrm{~min}$ the frequency was held at $1.94 \mathrm{MHz}$ and the power levels were varied at $18 \mathrm{~V}, 32 \mathrm{~V}$, and $52 \mathrm{~V}$ pk-pk. The results of this experiment showed a large increase in the thickness of oil layer on the emulsion when treated acoustically compared to the control and compared to the other experiments conducted.

\section{CONCLUSIONS}

Oil-water emulsions were made using mineral oil and 10W40 motor oil. Several transducer configurations were explored for producing standing waves in the emulsions. In all transducer geometries and configurations it was observed that oil droplets could be trapped and coalesced in standing waves produced to separate oil from emulsions. In a side-byside experiment, the acoustically stimulated sample showed a significant difference in oil separation compared to the control sample. In the case of motor oil-water emulsion, the contrast factor is low and the viscosity is significantly higher than that of mineral oil. However, it was still possible to separate this emulsion in simple resonant cavities without having to employ any porous media or other artifact in the cavity and at reasonable powers. Too much power would have induced both streaming and heating.

In regards to practical implementation of acoustic for liquid-liquid separation, direct contact of transducer to liquid was observed to be most effective in separating emulsions. Surface area in relation to volume appears to have a more dominant role rather than shape in effectively separating oil water emulsions, however shape can play a part in the practical implementation of acoustics for liquid-liquid separation. Variation of frequency, power, and wave function showed a positive effect in coalescing oil droplets in standing waves.

\section{ACKNOWLEDGEMENT}

Support for this research was provided by the Chevron Corporation through CRADA No. LA07C10518.

\section{REFERENCES}

[1] Whitworth G, Coakley WT. Particle column formation in a stationary ultrasonic field. J Acoust Soc Am 1992; 91: 79-85.

[2] Sollner K, Bondy C. The mechanism of coagulation by ultrasonic waves. Trans Faraday Soc 1926; 32: 616-23.

[3] Krishnan RS, Venkatasubramanian VS, Rajagopal ES. Effects of sound intensity on dispersion and coagulation in ultrasonic emulsification. Br J Appl Phys 1959; 10: 250-52.

[4] Gardner EA, Apfel RE. Using acoustics to study and stimulate the coalescence of oil drops surrounded by water. J Col Int Sci 1993; 159: 226-37.

[5] Harpur IG, Wayth NG, Bailey AG, Thew MT, Williams TJ, Urdahl O. Destablilisation of water-in-oil emulsions under the influence of an A.C. electric field: Experimental assessment of performance. J Electrost 1997; 40: 135-40.

[6] Pangu GD, Feke DL. Acoustically aided separation of oil droplets from aqueous emulsions. Chem Eng Sci 2004; 59: 3183-93.

[7] Crum LA. Acoustic force on a liquid droplet in an acoustic stationary wave. J Acoust Soc Am 1972; 50: 157-63.

[8] Stack JL, Carney PA, Malone HB, Wessels TK. Factor influencing the ultrasonic separation of oil in water emulsions. Ultra Sonochem 2005; 12: 153-60.

[9] King LV. On the acoustic radiation pressure on circular discs: inertia and diffraction corrections. Proc Royal Soc A 1935; 153.

[10] Yosioka K, Kawasima Y. Acoustic radiation pressure on a compressible sphere. Acustica 1955; 5: 167-73. 
[11] Ahuja AS, Hendee WR. Effects of particle shape and orientation on propagation of sound in suspensions. J Acoust Soc Am 1978; 63(4): 1074

[12] Weiser MAH, Apfel RE. Extension of acoustic levitation to include the study of micron-size particles in a more compressible host liquid. J Acoust Soc Am 1982; 71: 1261.

[13] Gor'Kov LP. On the forces acting on a small particle in an acoustical field in an ideal fluid. Soc Phys Doklady 1962; 6: 773.

[14] Told TL, Feke DL. Separation of dispersed phases from liquids in acoustically driven chambers. Chem Eng Sci 1953; 48(3): 521-40.

[15] Onda Corporation, Acoutistic properties of liquid, 2008, http://www.ondacorp.com/tables/Liquids.pdf, 2006

[16] Zheng X, Apfel RE. Acoustic interaction forces between two fluid spheres in an acoustic field. J Acoust Soc Am 1995; 97 (4): 221826.

[17] Pangu GD, Feke DL. Droplet transport and coalescence kinetics inemulsions subjected to acoustic fields. Ultrasonics 2007; 46: 289302
[18] Perales F, González I. On the forces acting in micromanipulation of particles at low frequencies. IEEE Ultra Symp 2005; 2108-11.

[19] Tuziuti T, Kozuka T, Mitome H. Measurement ofdistribution of acoustic radiation force perpendicular to sound beam axis. J Appl Phys 1999; 3: 3297-301.

[20] Spengler JF, Coakley WT, Christensen KT. Microstreaming effects on particle concentration in an Ultrasonic standing wave. AIChE J 2003; 49(11): 2773 .

[21] Kuznetsova LA, Coakley WT. Microparticle, concentration in short path length ultrasonic resonators: Roles of radiation pressure and acoustic streaming. J Acoust Soc Am 2004; 116(4): 1956-66.

[22] Sinha DN, Kaduchak G. Noninvasive Determination of Sound Speed and Attenuation in Chemical Warfare Agents in Elastic Properties of Fluids: Liquids and Gases. Academic Press 2001; IV: 3-21, Chapter 1.

[23] Meng G, Jaworski AJ, White NM. Composition measurements of cude oil and process water emulsions using thick-film ultrasonic transducers. Chem Eng Proc 2006; 45: 383-91.

Received: May 13, 2008

Revised: June 24, 2008

Accepted: July 30, 2008

(c) Garcia-Lopez and Sinha; Licensee Bentham Open.

This is an open access article licensed under the terms of the Creative Commons Attribution Non-Commercial License (http://creativecommons.org/licenses/by$\mathrm{nc} / 3.0 /$ ) which permits unrestricted, non-commercial use, distribution and reproduction in any medium, provided the work is properly cited. 